\title{
Phase-plane analysis of conserved higher-order traffic flow model *
}

\author{
Chun-xiu Wu $(\text { 吴春秀 })^{1,2} ， \quad$ Tao Song $(\text { 宋涛 })^{1,2}, \quad$ Peng Zhang(张鹏 $)^{1,2}, \quad$ S.C. Wong $(\text { 黄仕进 })^{3}$ \\ (1. Shanghai Institute of Applied Mathematics and Mechanics, Shanghai University, \\ Shanghai, 200072, P.R. China; \\ 2. Shanghai Key Laboratory of Mechanics in Energy Engineering, \\ Shanghai, 200072, P.R. China; \\ 3. Department of Civil Engineering, The University of Hong Kong, Pokfulam Road, \\ Hong Kong SAR, P.R. China)
}

\begin{abstract}
The phase-plane analysis is used to study the traveling wave solution of a recently proposed higher-order traffic flow model under the Lagrange coordinate system. The analysis identifies the types and stabilities of the equilibrium solutions, and the overall distribution structure of the nearby solutions is drawn in the phase plane for the further analysis and comparison. The analytical and numerical results are in agreement, and may help to explain the simulated phenomena, such as the stop-and-go wave and oscillation near a bottleneck. The findings demonstrate the model ability to describe the complexity of congested traffic.
\end{abstract}

Key words traffic flow; Lagrange coordinates; phase-plane analysis; traffic congestion patterns

\section{Introduction}

The instability of traffic flow has been studied for decades through observation and modeling, among which stop-and-go waves (especially jams) are currently an important subject. To depict instabilities that are characterized by oscillations in congested traffic, time series loop data in a certain location are often used for analysis in the flow-density phase plane. Usually, the stop-and-go wave embodies wide moving jams, which constitute a stable non-trivial solution and are distinguished from constant equilibrium traffic states. The phenomenon could be analytically described by studying traveling waves (including a shock) in most current higher-order models under periodic boundary conditions [1-7]. However, there are some extended patterns of congested traffic, which appear mainly in the upstream of a bottleneck with a fixed downstream wave front. Helbing [8] demonstrated two typical patterns of such congested traffic: oscillatory congested traffic (OCT) and homogeneous congested traffic (HCT). He attributed these congested patterns to the changes in flow rates upstream the main road and ramp, according to a detailed analysis on the observed flow-density phase plots. It has been widely regarded that, given the same initial conditions, these congested patterns could be reproduced by varying the aforementioned flow rates (being taken as the boundary conditions). As an instance, the phenomena were reproduced by the macro- and micro- models in [9]. Nevertheless, unlike that for the wide moving jams, few analytical properties are known for the aforementioned OCT and $\mathrm{HCT}$ patterns.

The phase-plane analysis has proved a suited tool for analysis of complex traffic flow phenomena $[1,10-13]$. Kühne [10] predicted Hopf-bifurcation, solitary wave, and periodic oscillatory solutions in a viscid higher-order model, which may suggest bistability and help well explain

* Project supported by the National Natural Science Foundation of China (No. 11072141), Shanghai Program for Innovative Research Team in Universities, Graduate Innovation Foundation of Shanghai University (SHUCX101078), and the University Research Committee, HKU SPACE Research Fund and Faculty of Engineering Top-up Grant of the University of Hong Kong (No. 201007176059).

Corresponding author: Peng Zhang, Professor; E-mail: pzhang@mail.shu.edu.cn 
hysteresis in congested traffic. Kerner and Konhäuser [1], and Wilson and Berg [11], demonstrated similar phenomena in the same fashion. In [12], more details were presented based on a general viscid higher-order model, and the traveling wave was classified as seven types which are in accordance with the reported observations. Moreover, Lee et al. [12] explained the mechanism for the existence of a limit circle solution showed in the so called inertia car-following model [14]. More recently, Saavedra and Velasco [13] adopted numerical method for the study and the results were compared with the analysis.

The present paper adopts a similar theory to study a recently proposed conservative higherorder (CHO) traffic flow model [15]. Despite the consistency with the first-order LighthillWhitham-Richards (LWR) model $[16,17]$, this model was able to reproduce many complex phenomena, such as stop-and-go waves, oscillation near a bottleneck, and queuing and dissipation at a signal $[15,18]$. Thus, it is significant for further understanding and development of the model to apply the theory. We emphasize on the classification and stability of the equilibrium solutions which significantly influence the behavior of the nearby non-trivial traveling wave solutions. It is also remarkable that the stability of these equilibrium solutions is discussed in two directions under the Lagrange coordinate system, i.e., with the independent variable going to both positive and negative infinite. Helpfully, similar types of equilibrium solutions together with their stabilities are indicated; the numerical study is complemented to verify the analytical conclusions. This demonstrates that the $\mathrm{CHO}$ model is able to describe the complexity of congested traffic and may further help explain more complicated phenomena.

The remainder of the paper is organized as follows. In Section 2, we briefly discuss the model equations. In Section 3, the non-linear and the linearized systems for solving the traveling wave are derived, and the classification and stability of the equilibrium points in the systems are discussed. In Section 4, trajectories of numeral solutions near the equilibrium points are drawn in the phase plane for comparison with the analytical results. Section 5 concludes the paper.

\section{Model equations}

Anisotropic higher-order traffic flow models can be generally written in the following form:

$$
\begin{gathered}
\frac{\partial \rho}{\partial t}+\frac{\partial \rho u}{\partial x}=0 \\
\frac{\partial u}{\partial t}+u \frac{\partial u}{\partial x}=\frac{u_{e}(\rho)-u}{\tau}+c(\rho, u) \frac{\partial u}{\partial x}
\end{gathered}
$$

where $\rho(x, t)$ and $u(x, t)$ are the density and speed for solution, $\tau$ is the relaxation time, and $c(\rho, u)>0$ is the sonic speed. The two characteristic speeds of the model are easily indicated as $\lambda_{1}=u-c(\rho, u)$ and $\lambda_{2}=u$. If $c(\rho, u)$ only depends on the density $\rho$, denoted by $c(\rho, u)=\rho p^{\prime}(\rho)$ with $p(\rho)$ being the pressure, Eq. (1) is the Aw-Rascle [19] or Aw-Rascle-Zhang [20] model with relaxation.

Zhang et al. [15] introduced a "pseudo density" $w$, which is determined through $u=V(w)$, where $w \leqslant \rho$, and $V(\cdot)$ denotes a desired velocity-density relationship. In this case, the mass conservation of (1) can be rewritten as

$$
\frac{\partial \rho}{\partial t}+\frac{\partial \rho V(w)}{\partial x}=0
$$

Moreover, by assuming $c(\rho, u)=c(u)$, where $c(u)=-w V^{\prime}(w)$, the acceleration of (2) can be written as the following "pseudo mass conservation" equation:

$$
\frac{\partial w}{\partial t}+\frac{\partial w V(w)}{\partial x}=\frac{V(w)-u_{e}(\rho)}{\beta}
$$


Here, the parameter $\beta=-\tau V^{\prime}(w) \doteq \tau u_{f} / \rho_{\text {jam }}, \rho_{\text {jam }}$ is the maximal density, and $u_{f}$ is the free flow velocity. The system of (3) and (4) constitutes a so called conserved higher-order $(\mathrm{CHO})$ model under the Euler coordinate system. A remarkable feature of the $\mathrm{CHO}$ model is its consistency with the well-known LWR model, namely, Eq. (4) will be equivalent to Eq. (3) if we set $\rho=w$, and $u_{e}(\cdot)=V(\cdot)$.

Assume that $M(x, t)$ is the total mass not passing through position $x$ at time $t$. Then, $(M, T)$ constitutes a Lagrange coordinate system through the relations: $T=t, M_{x}(x, t)=\rho(x, t)$, and $M_{t}(x, t)=-\rho(x, t) u(x, t)$. For a solution variable $A(x, t) \equiv A(M, T)$, the first order derivatives in the model are transformed through the formulas:

$$
\frac{\partial A(M, T)}{\partial x}=\rho \frac{\partial A(M, T)}{\partial M}, \quad \frac{\partial A(M, T)}{\partial t}=-\rho u \frac{\partial A(M, T)}{\partial M}+\frac{\partial A(M, T)}{\partial T} .
$$

See [7] for reference. According to the transformation of (5), the system of (3) and (4) becomes

$$
\begin{gathered}
\frac{\partial s}{\partial t}-\frac{\partial V(w)}{\partial M}=0, \\
\frac{\partial s w}{\partial t}=\frac{s\left(V(w)-u_{e}(s)\right)}{\beta},
\end{gathered}
$$

where $s(M, T) \equiv 1 / \rho(x, t)$ is the specific volume, and we retrieve the time variable $t$ through $T=t$. Under the Lagrange coordinate system, the two characteristic speeds of the system can be easily indicated as $\lambda_{1}=w V^{\prime}(w) / s<0$, and $\lambda_{2}=0$, which clearly shows the anisotropy of the model.

In general, the inviscid system of (1) and (2) is characterized by weak or discontinuous solutions, thus the conservative form of (7) is essential. On the other hand, the phase-plane analysis is applicable only to smooth solutions, thus we add a viscous term to Eq. (7) to derive the following equation:

$$
\frac{\partial s w}{\partial t}=\frac{s\left(V(w)-u_{e}(s)\right)}{\beta}+\mu \frac{\partial^{2} w}{\partial M^{2}} .
$$

Equations (6) and (8) now constitute the viscid conserved higher-order (VCHO) model. We note that an adequately large viscous coefficient $\mu>0$ can help smooth a shock or discontinuity; moreover, it avoids the jump in velocity which unreasonably suggests an infinite traffic deceleration across the shock. However, the smoothness is unlikely to significantly change the solution profile of the original model in that the solution of a viscid model can sufficiently approach that of the corresponding inviscid model for $\mu \rightarrow 0$. See [5] for detailed discussion.

\section{Stability of equilibrium points in traveling wave solution}

Consider a traveling wave solution $s(\xi)$ and $w(\xi)$, where $\xi=M-c t$, and $c$ is the traveling wave. Substituting these for the solution variables of (6) and (8), we derive

$$
\begin{gathered}
-c \frac{d s}{d \xi}-\frac{d V(w)}{d \xi}=0, \\
-c \frac{d(s w)}{d \xi}=\frac{s\left(V(w)-u_{e}(s)\right)}{\beta}+\mu \frac{d^{2} w}{d \xi^{2}} .
\end{gathered}
$$

Integration of (9) over $\xi$ yields

$$
c s+V(w)=\text { const }=u_{*},
$$

where the parameter $u_{*}$ is actually the traveling wave under the Euler coordinate system. 
By applying Eq. (11), Eq. (10) turns out to be the following second-order ordinary differential (OD) equation for solving $w(\xi)$ :

$$
\frac{d^{2} w}{d \xi^{2}}+G\left(w, u_{*}\right) \frac{d w}{d \xi}+F\left(w, c, u_{*}\right)=0,
$$

with

$$
\begin{gathered}
G\left(w, u_{*}\right)=\frac{u_{*}-V(w)-w V^{\prime}(w)}{\mu} \\
F\left(w, c, u_{*}\right)=\frac{u_{*}-V(w)}{c \beta \mu}\left[V(w)-u_{e}\left(\frac{u_{*}-V(w)}{c}\right)\right] .
\end{gathered}
$$

We are now seeking for stable solutions of Eq. (12), which are physically meaningful with bounded limits for $\xi \rightarrow \pm \infty$. Here, the physics of Eq. (12) can be analogous to that for one dimensional motion of a particle with unit mass. Taken $\xi$ and $w$ as the temporal and spatial variables, Eq. (12) governs the motion of the particle in the force field $-F\left(w, c, u_{*}\right)$, where $G\left(w, u_{*}\right)$ denotes the damping coefficient, and $U\left(w, c, u_{*}\right)=\int^{w} F\left(w, c, u_{*}\right) d w$ denotes the potential. Let $y=d w / d \xi$, then Eq. (12) is transformed to the following first-order OD system:

$$
\left\{\begin{array}{l}
w^{\prime}=y, \\
y^{\prime}=-G\left(w, u_{*}\right) y-F\left(w, c, u_{*}\right) .
\end{array}\right.
$$

By setting the terms on the right hand side to be zero, we have $y=0$ and $F=0$, which determine the equilibrium points $\left(w_{i}, 0\right)$ of $(15)$. Further, let these terms be linearized through the Taylor expansion at $(w, y)=\left(w_{i}, 0\right)$. Then, we derive the linearized system of $(15)$ :

$$
\left\{\begin{array}{l}
w^{\prime}=y \\
y^{\prime}=-G\left(w_{i}, u_{*}\right) y-F^{\prime}\left(w_{i}, c, u_{*}\right)\left(w-w_{i}\right) .
\end{array}\right.
$$

The Jacobian characteristic equation of (16) reads:

$$
\lambda^{2}+G_{i} \lambda+F_{i}^{\prime}=0
$$

where we denote by $G_{i}=G\left(w_{i}, u_{*}\right)$, and $F_{i}^{\prime}=F^{\prime}\left(w_{i}, c, u_{*}\right)$.

The equilibrium point $\left(w_{i}, 0\right)$ is classified as (i) a saddle point for $F_{i}^{\prime}<0$; (ii) a nodal point for $G_{i}^{2}-4 F_{i}^{\prime}>0$, and $F_{i}^{\prime}>0$; (iii) a degenerate nodal point for $G_{i}^{2}-4 F_{i}^{\prime}=0$; (iv) a spiral point for $G_{i}^{2}-4 F_{i}^{\prime}<0$, and $G_{i} \neq 0$; and (v) a center for $F_{i}^{\prime}>0$, and $G_{i}=0$. According to the qualitative theory of the differential equations, system (16) is always unstable at a saddle point for $\xi \rightarrow \pm \infty$; it is stable at a nodal or spiral point for $\xi \rightarrow+\infty$ (or $\xi \rightarrow-\infty$ ), if $G_{i}>0$ (or $\left.G_{i}<0\right)$. That $d U / d w=F=0$, and $d^{2} U / d w^{2}=F^{\prime}>0$ at a nodal or spiral point suggests a locally minimal energy of $U$. This physically explains the aforementioned stability in that the damping effect should reduce the energy if the damping coefficient $G_{i}>0$ (or $G_{i}<0$ ), thus the "particle" tends to move toward the point for $\xi \rightarrow+\infty$ (or $\xi \rightarrow-\infty)$. Otherwise, the damping effects oppositely and suggests the instability.

We note that the nonlinear system of (15) shares the same equilibrium points with the linearized system of (16). Moreover, the two systems will be uniformly stable or unstable at these points if the real component of the characteristic values of (17) is non-zero, which is implied in the forthcoming discussion. The conclusion is due to the Hartman-Gorban linearization theorem. 


\section{Numerical simulation}

The two velocity-density relationships in the model are specified as follows $[1,18]$ :

$$
\begin{gathered}
u_{e}(s)=u_{f}\left[\left(1+\exp \left(\frac{l / s-0.25}{0.06}\right)\right)^{-1}-3.75 \times 10^{-6}\right] \\
V(w)=u_{f} \frac{1-w l}{\left[1+b w l+a(w l)^{2}\right]}
\end{gathered}
$$

where the length and free flow speed of a vehicle are taken as $l=4.5 \mathrm{~m}$, and $u_{f}=30 \mathrm{~m} / \mathrm{s}$, and the other two parameters are given by $a=4$, and $b=-0.8$. The other parameters (in Eqs. (4) and (8)) are taken as $\rho_{j a m}=1 / l, \mu=u_{f}$, and $\tau=3 s$. For convenience in the forthcoming discussion, the pseudo density $w$ and its derivative $y=d w / d \xi$ are scaled by $\rho_{j a m}, c$ by $\rho_{j a m} u_{f}$ and $u_{*}$ by $u_{f}$.

Table 1 Classification of the equilibrium points of system (16) with a number of model parameters, where $\Delta_{i}=G_{i}^{2}-4 F_{i}^{\prime}$.

\begin{tabular}{|c|c|c|c|}
\hline$\left(c, u_{*}\right)$ & $(-0.18,-0.35)$ & $(-0.18,-0.32)$ & $(-0.19,-0.35)$ \\
\hline \multirow{3}{*}{$w_{1}$} & $\begin{array}{c}=0.1764 \\
F_{i}^{\prime}<0, \text { saddle point } \\
\text { unstable for } \xi \rightarrow \pm \infty\end{array}$ & $\begin{array}{c}F_{i}^{\prime}<0.1850, \text { saddle point } \\
\text { unstable for } \xi \rightarrow \pm \infty\end{array}$ & $\begin{array}{c}F_{i}^{\prime}<0, \text { saddle point } \\
\text { unstable for } \xi \rightarrow \pm \infty\end{array}$ \\
\hline \multirow{2}{*}{$w_{2}$} & $\begin{array}{c}=0.6340 \\
\Delta_{i}<0, G_{i}>0, \text { spiral point } \\
\text { stable for } \xi \rightarrow+\infty, \\
\text { unstable for } \xi \rightarrow-\infty .\end{array}$ & $\begin{array}{c}\Delta_{i}<0, G_{i}>0, \text { spiral point } \\
\text { stable for } \xi \rightarrow+\infty, \\
\text { unstable for } \xi \rightarrow-\infty .\end{array}$ & $\begin{array}{c}\Delta_{i}<0, G_{i}<0, \text { spiral point } \\
\text { unstable for } \xi \rightarrow+\infty, \\
\text { stable for } \xi \rightarrow-\infty .\end{array}$ \\
\hline \multirow{2}{*}{$w_{3}$} & $\begin{array}{c}=0.9315 \\
F_{i}^{\prime}<0, \text { saddle point } \\
\text { unstable for } \xi \rightarrow \pm \infty\end{array}$ & $\begin{array}{c}=0.9737 \\
F_{i}^{\prime}<0, \text { saddle point } \\
\text { unstable for } \xi \rightarrow \pm \infty\end{array}$ & $\begin{array}{c}=0.9624 \\
F_{i}^{\prime}<0, \text { saddle point } \\
\text { unstable for } \xi \rightarrow \pm \infty\end{array}$ \\
\hline
\end{tabular}

A certain pair of values of $c$ and $u_{*}$ determines three equilibrium points of (16), which are represented by $w_{i}(i=1,2,3)$, and the types and stabilities of these points are shown in Table 1 according to the discussion Section 2. Here, we note that the choice of $u_{*}$ helps retrieve a traveling wave speed around $10 \mathrm{~m} / \mathrm{s}$, which is in accordance with the observed data. Since it is unlikely to derive the analytical solution of the nonlinear system of (15), numerical solutions with proper initial data are given to study the stabilities of the system at the equilibrium points, which agrees with the conclusion in Table 1, as indicated in Figs. 1-3.

(a)

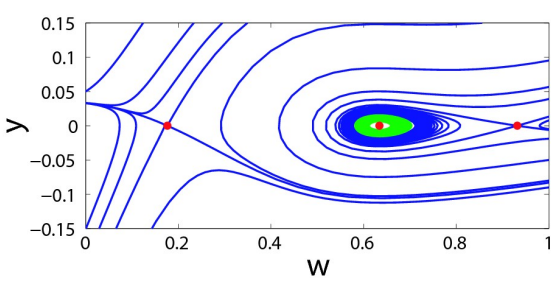

Fig. 1 (a) Trajectories in $w$ - $y$ phase plane; (b) evolutions of $w$ for $\xi \in[-2000,5000]$, with the initial data $(w(0), y(0))=(0.5817,0)$.

Fig. 1 corresponds to the first case in Table 1, which shows the instability of the system at the equilibrium points $\left(w_{1}, 0\right)$ and $\left(w_{3}, 0\right)$ for $\xi \rightarrow \pm \infty$, in that a nearby perturbed state would go far way (Fig. 1(a)). The area that encompasses the spiral point $\left(w_{2}, 0\right)$ represents a twisted trajectory starting from $(0.5817,0)$, which approaches the spiral point for $\xi \rightarrow+\infty$ and 
suggests the stability of the system at the spiral point for $\xi \rightarrow+\infty$. The system is unstable at $\left(w_{2}, 0\right)$ for $\xi \rightarrow-\infty$, the trajectory in this trend leaves the spiral point and eventually evolves into an oscillation with constant amplitude. Evolution of $w(\xi)$ along this trajectory is shown in Fig. 1(b). On the other hand, a twisted trajectory goes very closely to the aforementioned area outside for $\xi \rightarrow-\infty$; it goes to infinite for $\xi \rightarrow+\infty$ (Fig. 1(a)). Therefore, there must exist periodic a limit circle solution between the aforementioned two trajectories, and the solution in Fig. 1(b) can be considered as a limit circle-spiral point solution.

(a)

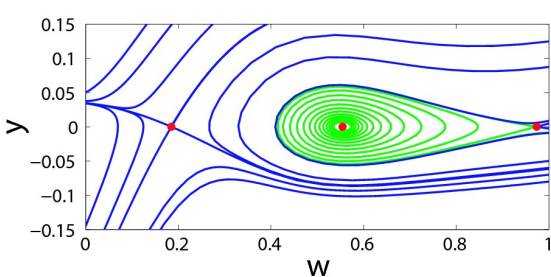

Fig. 2 (a) Trajectories in $w-y$ phase plane; (b) evolutions of $w$ for $\xi \in[0,1000]$, with the initial data $(w(0), y(0))=(0.97365,0)$.

Fig. 2 corresponds to the second case in Table 1, which similarly indicates the instability of the system at the equilibrium points $\left(w_{1}, 0\right)$ and $\left(w_{3}, 0\right)$ for $\xi \rightarrow \pm \infty$ (Fig. 2(a)). The spiral point $\left(w_{2}, 0\right)$ is encompassed and approached by a twisted curve for $\xi \rightarrow+\infty$, which suggests the stability of the system at this point in the trend. In the other trend, the curve travels very close to $\left(w_{3}, 0\right)$ but eventually goes to infinite, which suggests the instability of the system at $\left(w_{2}, 0\right)$ for $\xi \rightarrow-\infty$. See also the evolution of $w(\xi)$ along this trajectory in Fig. 2(b). We note that similar curves can be drawn among which the one passing through $\left(w_{2}, 0\right)$ is known as a saddle-spiral point solution.

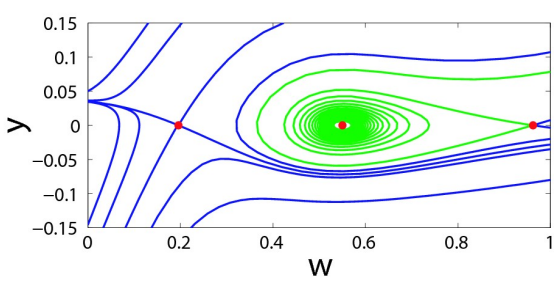

(a)

Fig. 3 (a) Trajectories in $w-y$ phase plane; (b) evolutions of $w$ for $\xi \in[-1000,100]$, with the initial data $(w(0), y(0))=(0.96,0)$.

Fig. 3 corresponds to the third case in Table 1. We again observe the instability of the system at $\left(w_{1}, 0\right)$ and $\left(w_{3}, 0\right)$. However, the curve that is shown to encompass the spiral point $\left(w_{2}, 0\right)$ approaches and leaves away from this point respectively for $\xi \rightarrow-\infty$ and $\xi \rightarrow+\infty$. This suggests that the system is stable and unstable at $\left(w_{2}, 0\right)$ respectively for $\xi \rightarrow-\infty$ and $\xi \rightarrow+\infty$. See also Fig. 3(b) for comparison with Fig. 2(b), and similar curves could be drawn to indicate the same stability or instability at $\left(w_{2}, 0\right)$, among which there exists a saddle-spiral point solution.

\section{Conclusions}

We have studied the traveling wave solution of the VCHO model based on the phase-plane analysis, which helps classify the three equilibrium points of the model, and thus indicates the stability or instability of the system at these points. Numerical solution gives an overall 
structure of trajectories in the phase plane, which is sensitive to the model parameters. It is significant to observe limit circle, limit circle-spiral point, and saddle-spiral point solutions, which should be useful for a better understanding of many complex phenomena in congested traffic.

Nevertheless, many have remained unknown for the discussed system or the like. For instance, two varying parameters $c$ and $u_{*}$ should pose more difficulty and complexity for the discussion based on the bifurcation theory.

\section{References}

[1] Kerner, B.S. and Konhäuser, P. Structure and parameters of clusters in traffic flow. Physical Review E, 50, 54-83 (1994).

[2] Greenberg, J.M. Congestion redux. SIAM Journal on Applied Mathematics, 64(4), 1175-1185 (2004).

[3] Siebel, F. and Mauser, W. Synchronized flow and wide moving jams from balanced vehicular traffic. Physical Review E, 73, 066108 (2006).

[4] Zhang, P., Wong, S. C., and Dai, S. Q. Characteristic parameters of a wide cluster in a higher-order traffic flow model. Chinese Physics Letters, 23, 516-519 (2006).

[5] Zhang, P. and Wong, S.C. Essence of conservation forms in the traveling wave solutions of HigherOrder Traffic Flow Model. Physical Review E, 74, 026109 (2006).

[6] Xu, R.Y., Zhang, P., Dai, S.Q., and Wong, S.C. Admissiblity of a wide cluster solution in "anisotropic" higher-order traffic flow models. SIAM Journal on Applied Mathematics, 68, 562-573 (2007).

[7] Zhang, P., Wu, C.X., and Wong, S.C. A semi-discrete model and its approach to a solution for wide moving jam in traffic flow. Physica A, 391, 456-463 (2012).

[8] Helbing, D., Hennecke, A., and Treiber, M. Phase Diagram of Traffic States in the Presence of Inhomogeneities. Physical Review Letters, 82, 4360 (1999).

[9] Treiber, M., Kesting, A. and Helbing, D. Three-phase traffic theory and two-phase models with a fundamental diagram in the light of empirical stylized facts. Transportation Research Part B, 44, 983-1000 (2010).

[10] Kühne, R.D. Traffic patterns in unstable traffic flow on freeways. In: Highway capacity and level of service, Proceedings of the International Symposium on Highway Capacity (ed. U. Brannolte), Balkema, Rotterdam, 211-223 (1991).

[11] Wilson, R.E. and Berg, P. Existence and classification of traveling wave solutions to second order highway traffic models. in Traffic and Granular Flow' 01 (eds. M. Fukui, Y. Sugiyama, M. Schreckenberg, and D. E. Wolf,), Springer, Berlin, 85-90 (2003).

[12] Lee, H.K., Lee, H.-W., and Kim, D. Steady-state solutions of hydrodynamic traffic models. Physical Review E, 69, 016118 (2004).

[13] Saavedra, P. and Velasco, R.M. Phase-space analysis for hydrodynamic traffic models. Physical Review E, 79, 066103 (2009).

[14] Tomer, E., Safonov, L., Havlin, and S. Presence of many stable nonhomogeneous states in an inertial car-following model. Physical Review Letters, 84, 382-385 (2000).

[15] Zhang, P., Wong, S.C., and Dai, S.Q. A conserved higher-order anisotropic traffic flow model: Description of equilibrium and non-equilibrium flows. Transportation Research Part B, 43, 562574 (2009).

[16] Lighthill, M.J. and Whitham, G.B. On kinematic waves: II. A theory of traffic flow on long crowded roads. Procrrdings of the Royal Society of London, Series A, 229(1178), 317-345 (1955).

[17] Richards, P.I. Shockwaves on the highway. Operations Research, 4(1), 42-51 (1956).

[18] Zhang, P., Qiao, D.L., Dong, L.Y., Dai, S.Q., and Wong,S.C. A number of Riemann solvers for a conserved higher-order traffic flow model. the Fourth International Joint Conference on Computational Sciences and Optimization, 1049-1053 (2011). 
[19] Rascle, M. An improved macroscopic model of traffic flow: Derivation and links with the LighthillWhitham model. Mathematical and Computer Modelling, 35, 581-590 (2002).

[20] Zhang, H.M. A non-equilibrium traffic model devoid of gas-like behavior. Transportation Research Part B, 36, 275-290 (2002). 